\title{
Percutaneous Transforaminal Epidural Injection Method in an Experimental Rat: Minimally Invasive Drug Delivery Method to Spinal Epidural Space
}

\author{
Nack Hwan Kim, M.D., Sang Heon Lee, M.D., Ph.D. ${ }^{1}$, Seok Jun Lee, M.D. ${ }^{1}$ \\ Department of Physical Medicine \& Rehabilitation, Gunsan Medical Center, Gunsan 573-713, \\ ${ }^{1}$ Department of Physical Medicine \& Rehabilitation, Korea University College of Medicine, Seoul 136-705, Korea
}

\begin{abstract}
Objective To compare a newly developed minimally-invasive method for percutaneous transforaminal epidural injection (INJ group) with the existing method for lumbar epidural catheterization (CATH group).

Method Through anatomical review of experimental rats, the cephalic one fourth of the neural foramen was selected as the target point for drug delivery. After the rats had undergone lumbar epidural catheterization, lidocaine, and $1 \%$ methylene blue were injected through the unilateral or bilateral L5/6 neural foramen in the INJ group, and through an epidural catheter in the CATH group. Measurement of body weight and the mechanical allodynia test before and after injection of lidocaine, and fine dissection after injection were performed.

Results Results of the mechanical allodynia test of $1.0 \%$ lidocaine $50 \mu$ injection in the CATH group were statistically similar to those of $0.5 \%$ lidocaine $100 \mu$ injection in the INJ group. The results of $2.0 \%$ lidocaine 50 $\mu \mathrm{l}$ injection in the CATH group were statistically similar to those of $1.0 \%$ lidocaine $100 \mu$ injection in the INJ group. After dissection, only one distal partial spinal nerve was stained by methylene blue $50 \mu \mathrm{l}$ through the transforaminal pathway. However, the dorsal root ganglion, nerve root, and adjacent hemi-partial spinal cord were stained by methylene blue $100 \mu$ through the transforaminal pathway.

Conclusion The percutaneous transforaminal epidural injection is practical, easy, and safe, and, in particular, does not cause significant pain compared to the existing lumbar epidural catheterization. We expect this method to be effective in an animal study showing that drug delivery to the spinal epidural space is necessary.
\end{abstract}

Key Words Epidural catheterization, Transforaminal, Animal study, Pain, Lidocaine

Received May 9, 2012; Accepted July 19, 2012

Corresponding author: Seok Jun Lee

Department of Physical Medicine \& Rehabilitation, Korea University College of Medicine, 73 Inchon-ro, Seongbuk-gu, Seoul 136-705, Korea Tel: +82-2-920-6471, Fax: +82-2-929-9951, E-mail: ljooll@korea.ac.kr

(c) This is an open-access article distributed under the terms of the Creative Commons Attribution Non-Commercial License (http:// creativecommons.org/licenses/by-nc/3.0) which permits unrestricted noncommercial use, distribution, and reproduction in any medium, provided the original work is properly cited.

Copyright $\odot 2012$ by Korean Academy of Rehabilitation Medicine

\section{INTRODUCTION}

Epidural catheterization is used widely as a reliable standard method for epidural drug delivery in experimental animals. It is also used for assessment of drug efficacy for clinical trials or the propriety of a pain-related animal model. ${ }^{1}$ However, this has significant disadvantages in animal studies of 'pain'. In order to secure ob- 
jectivity and significance, it is usually necessary to use a large sample of experimental animals. ${ }^{2}$ Considering cost, time, and labor of experimenters, this is an inefficient preparation procedure. ${ }^{3}$ Furthermore, development of fibrosis at the distal tip of the catheter occurs frequently, and the inserted catheter may irritate and inflame the thecal sac, an important factor in alteration of the pain threshold. ${ }^{4}$ In addition, time to recovery from the postsurgical condition is lengthy, and surgical injury develops in intervertebral ligaments, lamina, and adjacent soft tissues. A new method for delivery of drugs to the epidural space, which can remedy the above difficulties, is required for development of experimental efficacy. The purpose of this study is to compare the efficacy of lidocaine injected through the existing lumbar epidural catheter using a newly developed method for percutaneous transforaminal epidural injection.

\section{MATERIALS AND METHODS}

This study protocol was approved by our institutional animal care and use committee.

\section{Anatomical review in lumbar vertebrae of experimental animal cadavers}

Fine dissection of Sprague-Dawley rat cadavers was carried out. On a view of the lateral lumbar intervertebral foramen, the foramen was placed on the ventrolateral portion of the vertebral column, and the spinal nerve passed through the caudal portion of the foramen and

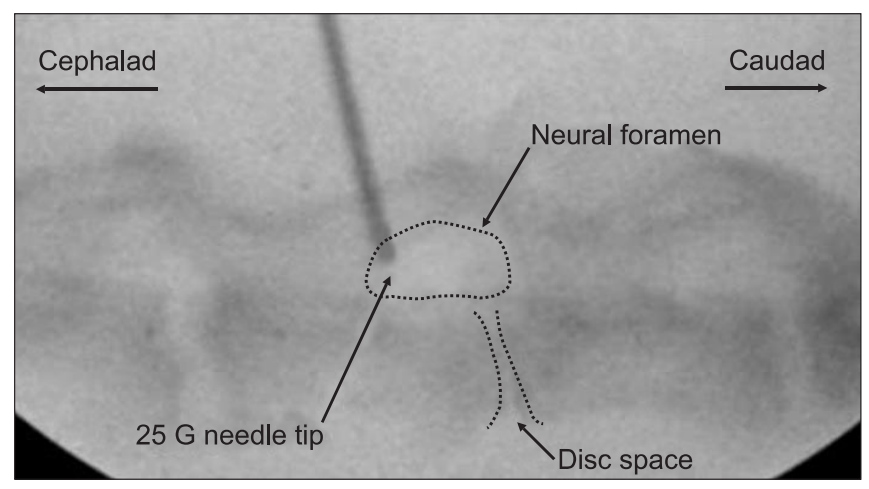

Fig. 1. Anatomic review of the lumbar spine of SpragueDawley cadavers. If the injected 25 gauge needle is approached to the cephalic area of the foramen circle on the lateral view of X-ray, injury to neural tissues placed on the caudal area of the foramen circle can be prevented. the medial side of the transverse process, and occupied a caudal quarter part of the foramen. On the lateral view using an X-ray generator OEC 9800 Plus (GE Medical System, Salt Lake City, USA), the vertebral column, intervertebral space, and foramen were well-demarcated, and the diameter of the foramen was three or four times that of the 25 gauge needle used commonly in clinics (Fig. 1). By X-ray image guidance the cephalic quarter portion of the foramen was selected as the target point of percutaneous transforaminal epidural injection.

\section{Experimental animals}

Eighty Sprague-Dawley rats 250-300 g (Central Lab Animal Inc., Seoul, Korea) underwent surgery for lumbar epidural catheterization. They were classified into two groups of epidural catheter injection (CATH) and transforaminal injection (INJ). Epidural drug administration was achieved through the epidural catheter in CATH group and transforaminal injection in INJ group. All procedures were done under anesthesia with halothane $4 \%$ in an anesthesia induction box, followed by continuous anesthetization with halothane $2 \%$ in an oxygen mixture with spontaneous respiration. An additional five rats without epidural catheterization were availed as a control group in the body weight measurement study (Fig. 2).

\section{Epidural catheterization}

The epidural catheter was made of a polyethylene tube having a length of $12 \mathrm{~cm}$ and an outer diameter of 0.61 $\mathrm{mm}$, in which the dead space was $8 \pm 1 \mu \mathrm{l}$. A knot was made at the distal $2 \mathrm{~cm}$ portion, and whether the tube

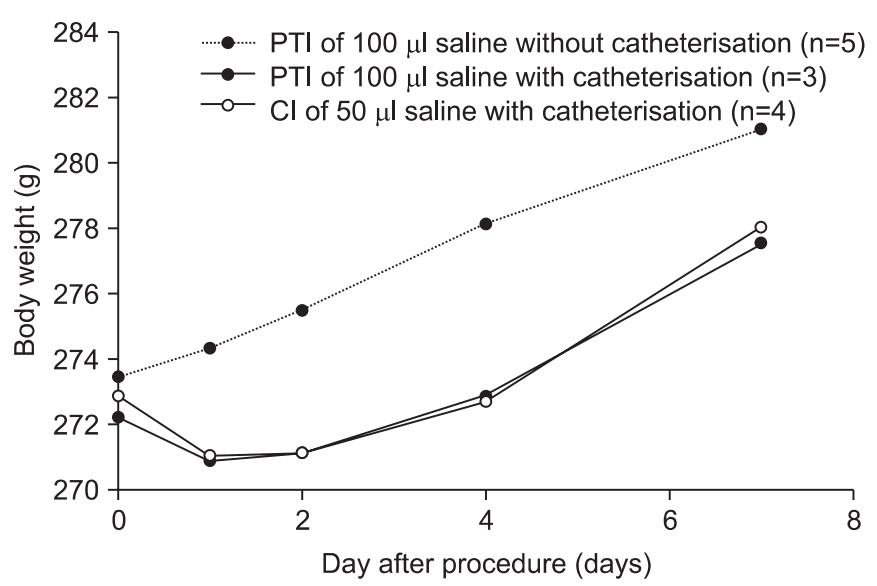

Fig. 2. Body weight changes of experimental rats. PTI: Percutaneous transforaminal injection, CI: Epidural catheter injection. 
was obstructed was confirmed by saline infusion.

For epidural catheterization, the dorsal thoracolumbar spinal region was sterilized with betadine and alcohol, and a $2 \mathrm{~cm}$ sagittal skin incision was made at the T13 spinous process. Muscle and fascia were dissected and retracted to expose the intervertebral ligament, which was carefully cut, and the catheter was inserted into the epidural space $2 \mathrm{~cm}$ caudally. The catheter tip was located at the L5/6 level, and the knot was placed in the space between the two adjacent vertebrae. The catheter was flushed with sterile saline, and no leakage was observed. The tip was plugged with a $28 \mathrm{G}$ short steel wire. The muscle, fascia, and skin were then sutured and sterilized. Inhalation of anesthetics was stopped and the rats were observed during recovery in a warm box. Rats with motor disturbance after 24 hours were excluded from this study. All animals were sacrificed with an over-dose of halothane after the study, and the spinal column was dissected with a catheter to verify the position of the tip. Rats with the tip not in the epidural space were excluded from this study.

\section{Percutaneous transforaminal epidural injections}

A needle with a 25 gauge and $1 \mathrm{~cm}$ length, commonly used in clinics, was used for procedure. Rats were anesthetized with halothane $2 \%$ in an air-oxygen mixture in an anesthesia induction box. In the lateral decubitus position, the L5/6 foramen was detected under X-ray image guidance. The target point of the needle tip was a cephalic quarter portion of the foramen, and the needle entry point was approximately $1 \mathrm{~cm}$ lateral to the midline at L5/6 dorsal skin. The needle was inserted and observed under X-ray image guidance. After injection, no bleeding or hematoma was observed during recovery.

\section{Body weight measurement}

Body weight was measured before, and at 1, 2, 4, and 7 days after procedure in three groups; percutaneous transforaminal injection of $100 \mu \mathrm{l}$ saline without catheterization $(n=5)$, percutaneous transforaminal injection of 100 $\mu \mathrm{l}$ saline with catheterization $(\mathrm{n}=3)$, and catheter injection of 50uL saline with catheterization $(n=4)$. The latter two groups with catheterization were provided from the control set groups at the following drug delivery study.

\section{Drugs delivery}

$0.5 \%, 1.0 \%$, and $2.0 \%$ Lidocaine (Huons, Seongnam, Korea) and sterilized saline $(0.9 \% \mathrm{NaCl})$ were administered. Animals in the control group were injected with $0.5 \%$, $1.0 \%$, and $2.0 \%$ lidocaine $50 \mu \mathrm{l}$ or saline $50 \mu \mathrm{l}$ through an epidural catheter over one minute by manual infusion (CATH group), and those in the experimental group were injected with $0.5 \%, 1.0 \%$, and $2.0 \%$ lidocaine 50 or $100 \mu \mathrm{l}$, or saline 50 or $100 \mu \mathrm{l}$ unilaterally or bilaterally by manual infusion over one minute (INJ group).

\section{Behavioral tests}

Behavioral testing for mechanical allodynia in the CATH and INJ groups was performed before and after administration of lidocaine or saline. ${ }^{5}$ Mechanical allodynia was assessed by the hindpaw withdrawal threshold in response to probing with a series of calibrated von Frey filaments (3.92, 5.88, 9.80, 19.60, 39.20, 58.80, 78.40, and $147.00 \mathrm{mN}$ [equivalent in grams to $0.4,0.6,1.0,2.0,4.0$, 6.0, 8.0, and 15.0, respectively]) (Stoelting, Wood Dale, USA). The $50 \%$ withdrawal threshold was determined using the up-down method. Tests were performed before, and $10,15,20,30,60,90$, and 120 minutes after drug administration.

\section{Methylene blue distribution test}

Prior to sacrifice of rats for completion of the experiment, four rats in the CATH group, which were injected with saline $50 \mu \mathrm{l}$, and another five rats without epidural catheterization which were set as a control in body weight measurement study were injected with methylene blue 50 or $100 \mu$ l through the epidural catheter or transforaminal pathway. After 12 hours, the rats were sacrificed and finely dissected for observation of the distribution of methylene blue in the epidural space. ${ }^{6}$

\section{Data analysis and statistics}

Data from behavioral tests were converted to \%MPE (maximal potential efficacy) according to the following formula:

\%MPE $=[($ post-drug threshold - base-line threshold $) /$ (cut off threshold - base-line threshold)] $\times 100$.

Statistical analysis was performed using Fisher's exact test. Null hypotheses of no difference were rejected if $p$ values were less than 0.05 . 


\section{RESULTS}

\section{Success rate of epidural catheterization}

Twelve (15.0\%) of eighty rats were excluded from the study. In seven rats $(8.8 \%)$, catheters were placed in the subarachnoid space, or penetrated the spinal cord. Four rats $(5.0 \%)$ were found with a removed catheter, and one rat (1.3\%) died just after administration of $2.0 \%$ lidocaine $50 \mu \mathrm{l}$ (Table 1).

\section{Body weight}

The weight of rats with percutaneous transforaminal epidural injection of $100 \mu \mathrm{l}$ saline without catheterization $(\mathrm{n}=5)$ increased progressively. Rats injected $50 \mu \mathrm{l}$ saline through lumbar epidural catheter $(n=4)$ lost weight one day after surgery and followed by weight gain, which were also similar to that of rats with percutaneous transforaminal epidural injection of $100 \mu \mathrm{l}$ saline with catheterization ( $n=3)$ (Fig. 2).

\section{Behavioral tests}

Effects of lidocaine with different concentrations with same $50 \mu$ in the CATH and INJ groups: The Mechanical allodynia test in the CATH and INJ groups was performed after administration of $0.5 \%, 1.0 \%$, and $2.0 \%$ lidocaine $50 \mu \mathrm{l}$ (L250, L500, and L1000). Unlike the INJ group, the CATH group results differed significantly corresponding to increments of concentration of lidocaine $(\mathrm{p}<0.05)$.
For determination of volume effects on injected epidural space, normal saline $50 \mu \mathrm{l}$ was applied in both groups and no notable behavioral changes were observed (Fig. 3-A, B).

Effects of lidocaine $50 \mu \mathrm{l}$ or $100 \mu \mathrm{l}$ in the INJ group: Transforaminal epidural injections of lidocaine differing in volume $(50 \mu \mathrm{l}$ and $100 \mu \mathrm{l})$ were performed. With lidocaine $50 \mu \mathrm{l}$, there was no change in behavioral test with difference of lidocaine concentration. However, with lidocaine $100 \mu \mathrm{l}$, the difference was significant according to the concentration $(\mathrm{p}<0.05)$ (Fig. $3-\mathrm{C})$.

Effects of unilateral and bilateral injection of lidocaine in the INJ group: The effect on the contralateral limb of rats injected on the unilateral side was assessed. Lidocaine $100 \mu \mathrm{l}$ at $0.5 \%, 1.0 \%$, and $2.0 \%$ was injected into the unilateral L5/6 foramen, and mechanical allodynia tests were taken on ipsilateral and contralateral hindpaws. With $0.5 \%$ and $1.0 \%$ lidocaine, tests of the contralateral hindpaw showed no significant change between before and after injection. With $2.0 \%$ lidocaine, however, the tests revealed significant changes. With all concentrations of lidocaine, significant differences were observed between ipsilateral and contralateral hindpaws after injection $(p<0.05)$. Tests of the ipsilateral hindpaw after unilateral or bilateral lidocaine injection $(100 \mu \mathrm{l}$ unilaterally, $100 \mu \mathrm{l}$ each bilaterally) showed no statistically significant difference with $0.5 \%$ and $1.0 \%$ lidocaine. A statistically significant difference was observed with

Table 1. Distribution of Experimental Animals

\begin{tabular}{ccccc}
\hline \multirow{2}{*}{$\begin{array}{c}\text { CATH group } \\
(\mathbf{n}=\mathbf{1 7})\end{array}$} & \multicolumn{2}{c}{ Unilateral injection } & \multicolumn{2}{c}{ Bilateral injection } \\
\cline { 2 - 5 } $0.5 \%$ Lidocaine & $0.5 \% \mathrm{Lidocaine}$ & $0.5 \%$ Lidocaine & $0.5 \%$ Lidocaine & $0.5 \%$ Lidocaine \\
$50 \mu \mathrm{l}$ & $50 \mu \mathrm{l}$ & $100 \mu \mathrm{l}$ & $50 / 50 \mu \mathrm{l}$ & $100 / 100 \mu \mathrm{l}$ \\
$(\mathrm{L} 250, \mathrm{n}=4)$ & $(\mathrm{L} 250, \mathrm{n}=4)$ & $(\mathrm{L} 500, \mathrm{n}=3)$ & $(\mathrm{L} 250 / 250, \mathrm{n}=4)$ & $(\mathrm{L} 500 / 500, \mathrm{n}=3)$ \\
$1.0 \%$ Lidocaine & $1.0 \%$ Lidocaine & $1.0 \%$ Lidocaine & $1.0 \%$ Lidocaine & $1.0 \%$ Lidocaine \\
$50 \mu \mathrm{l}$ & $50 \mu \mathrm{l}$ & $100 \mu \mathrm{l}$ & $50 / 50 \mu \mathrm{l}$ & $100 / 100 \mu \mathrm{l}$ \\
$(\mathrm{L} 500, \mathrm{n}=5)$ & $(\mathrm{L} 500, \mathrm{n}=3)$ & $(\mathrm{L} 1000, \mathrm{n}=4)$ & $(\mathrm{L} 500 / 500, \mathrm{n}=3)$ & $(\mathrm{L} 1000 / 1000, \mathrm{n}=4)$ \\
$2.0 \% \mathrm{Lidocaine}$ & $2.0 \% \mathrm{Lidocaine}$ & $2.0 \% \mathrm{Lidocaine}$ & $2.0 \%$ Lidocaine & $2.0 \%$ Lidocaine \\
$50 \mu \mathrm{l}$ & $50 \mu \mathrm{l}$ & $100 \mu \mathrm{l}$ & $50 / 50 \mu \mathrm{l}$ & $100 / 100 \mu \mathrm{l}$ \\
$(\mathrm{L} 1000, \mathrm{n}=4)$ & $(\mathrm{L} 1000, \mathrm{n}=3)$ & $(\mathrm{L} 2000, \mathrm{n}=4)$ & $(\mathrm{L} 1000 / 1000, \mathrm{n}=3)$ & $(\mathrm{L} 2000 / 2000, \mathrm{n}=3)$ \\
Saline & Saline & Saline & Saline & Saline \\
$50 \mu \mathrm{l}$ & $50 \mu \mathrm{l}$ & $100 \mu \mathrm{l}$ & $50 / 50 \mu \mathrm{l}$ & $100 / 100 \mu \mathrm{l}$ \\
$(\mathrm{S} 50, \mathrm{n}=4)$ & $(\mathrm{S} 50, \mathrm{n}=2)$ & $(\mathrm{S} 50, \mathrm{n}=3)$ & $(\mathrm{S} 50 / 50, \mathrm{n}=2)$ & $(\mathrm{S} 100 / 100, \mathrm{n}=3)$ \\
\hline
\end{tabular}

Twelve of eighty individuals were excluded from the experiment, because of catheter placed on subarachnoid space, catheter-related spinal cord injury, catheter removal during experiment, and anaphylactic shock due to lidocaine CATH group: Lumbar epidural catheter injection group, INJ group: Transforaminal epidural injection group 

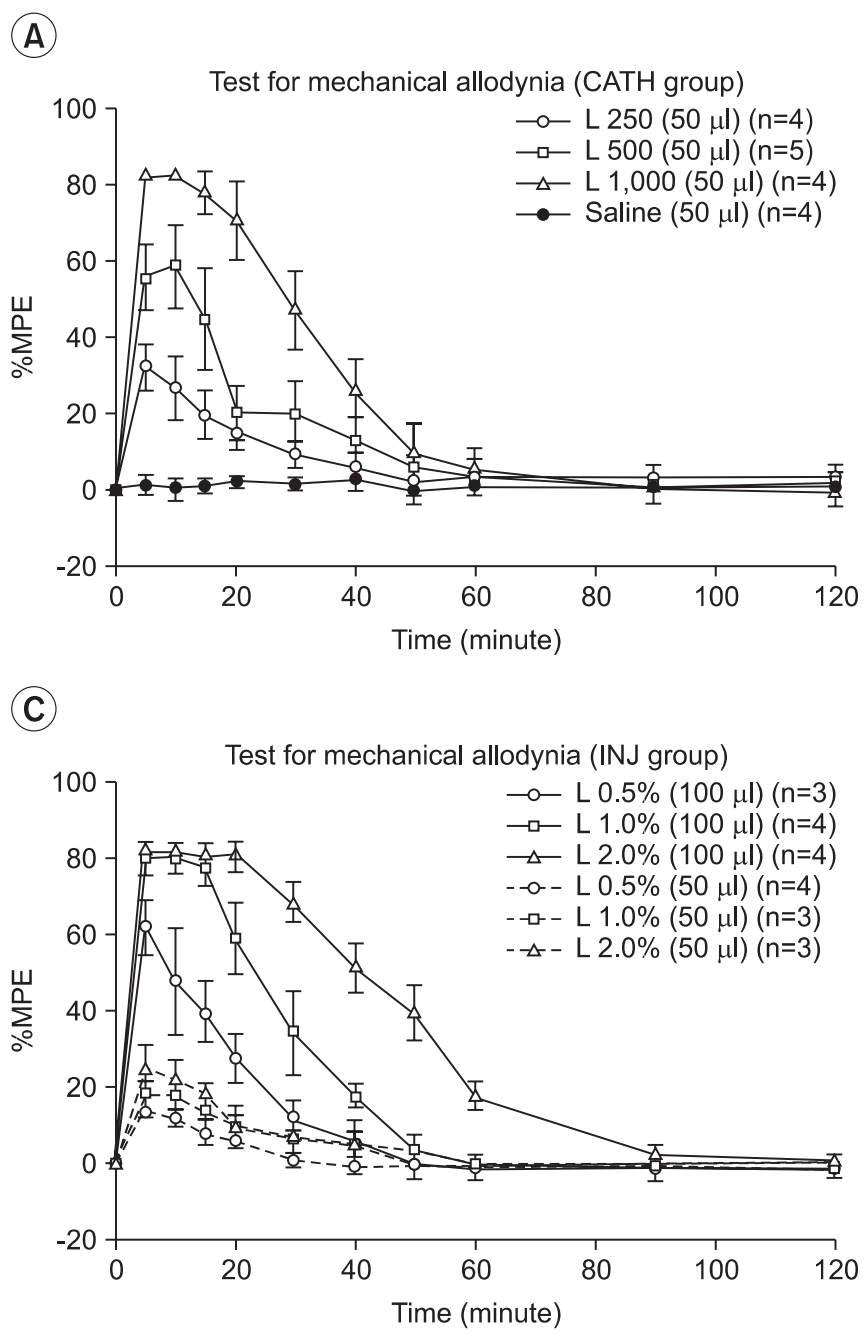

$2.0 \%$ lidocaine $(\mathrm{p}<0.05)$ (Fig. 4-A, B).

Comparison of effects between epidural catheterization and percutaneous transforaminal epidural injection: Drug potential efficacy of $1.0 \%$ lidocaine $50 \mu \mathrm{l}$ injected through an epidural catheter was did not differ statistically from that of $0.5 \%$ lidocaine $100 \mu \mathrm{l}$ injected through a unilateral or bilateral percutaneous transforaminal pathway. Drug potential efficacy of $2.0 \%$ lidocaine $50 \mu$ injected through an epidural catheter did not differ statistically from that of $1.0 \%$ lidocaine $100 \mu \mathrm{l}$ injected through a unilateral or bilateral percutaneous transforaminal pathway (Fig. 4-C).

\section{Epidural distribution of methylene blue}

Epidurally injected methylene blue $50 \mu \mathrm{l}$ was distributed to two or three segments of the bilateral thecal sac in the CATH group, and distal spinal nerve and adjacent soft tissues in the INJ group. Epidurally injected methylene

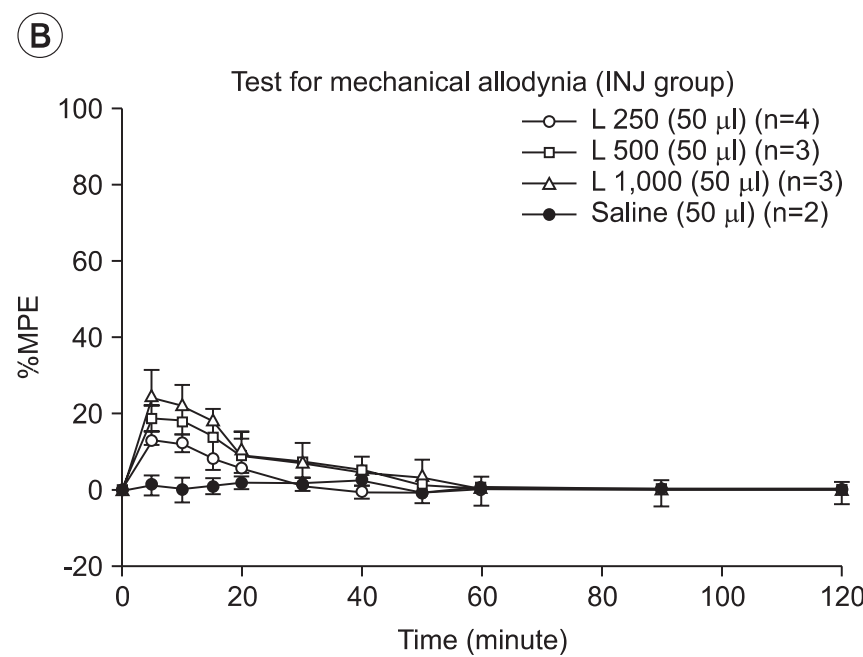

Fig. 3. Results of the mechanical allodynia test in the CATH and INJ groups. (A) $0.5 \%, 1.0 \%$, and $2.0 \%$ lidocaine $50 \mu \mathrm{l}$ injection in the CATH group. (B) $0.5 \%, 1.0 \%$, and $2.0 \%$ lidocaine $50 \mu \mathrm{l}$ injection in the INJ group. (C) Comparison between the results of lidocaine $50 \mu \mathrm{l}$ and $100 \mu \mathrm{l}$ injection in the INJ group.

blue $100 \mu \mathrm{l}$ was distributed to one or two segments of the unilateral thecal sac, ipsilateral adjacent spinal root, and dorsal root ganglion on the same level in the INJ group (Fig. 5).

\section{DISCUSSION}

A new method of percutaneous transforaminal epidural injection in experimental rats without dissection of the muscle or ligament, and catheter implantation has been developed. This method has a much lower failure rate for delivery of drugs to the epidural space than catheterization, and is very efficient in regard to time, cost, and labor. The percutaneous injection takes less than one minute, with no recovery period, and can even be easily performed by a beginner. This method is already used for patients with spinal radiculopathy or spinal stenosis, and can be performed safely by an experienced physician un- 
(A)

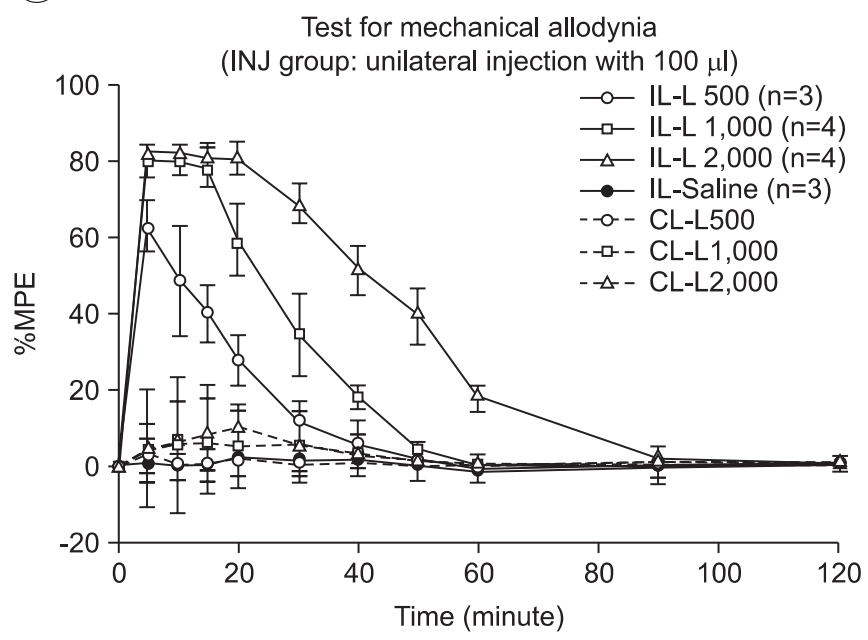

(C)

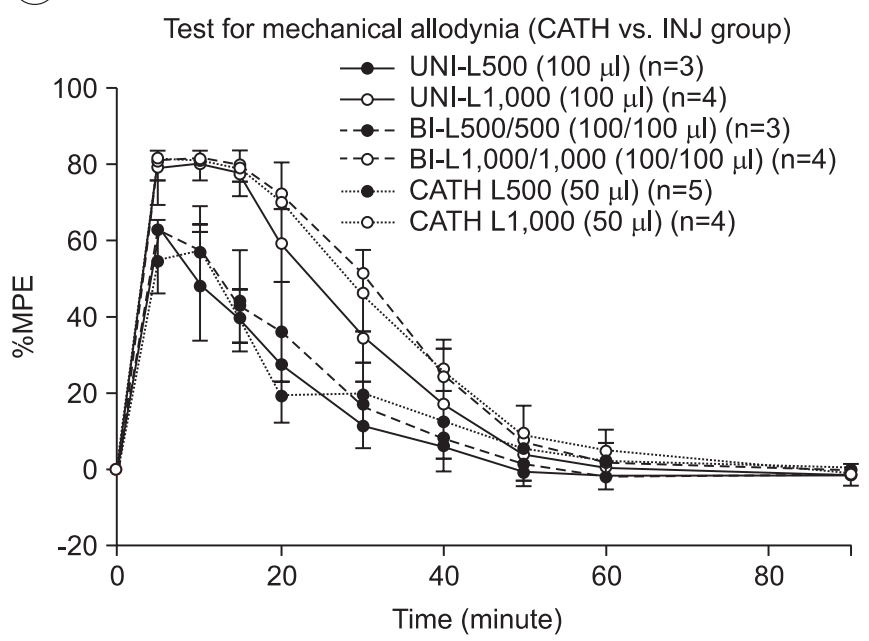

der C-arm guidance. ${ }^{7}$

The operation for epidural catheterization has many problems. It takes at least 10 minutes, and the recovery period after surgery is approximately 24 hours. ${ }^{2}$ Careful supervision for the likelihood of infection on the operation site and prevention of removal of the catheter by animals, was labor-intensive to experimenters. ${ }^{8}$ An indwelling catheter may distort the assessment of pain threshold due to irritation or mechanical injury to the thecal sac, spinal cord, and nerves, ${ }^{9}$ and, if obstruction or deformation occur, the resulting data would contain a large error. ${ }^{10}$ In addition, fibrosis is a common complication, and obstructs the catheter or interferes with absorption of drugs into dura. ${ }^{11}$ The failure rate of epidural catheter placement was $15.0 \%$ in this study.

Catheterization has a considerable effect on the pain threshold. Quantitative pain assessment in experimental
(B)

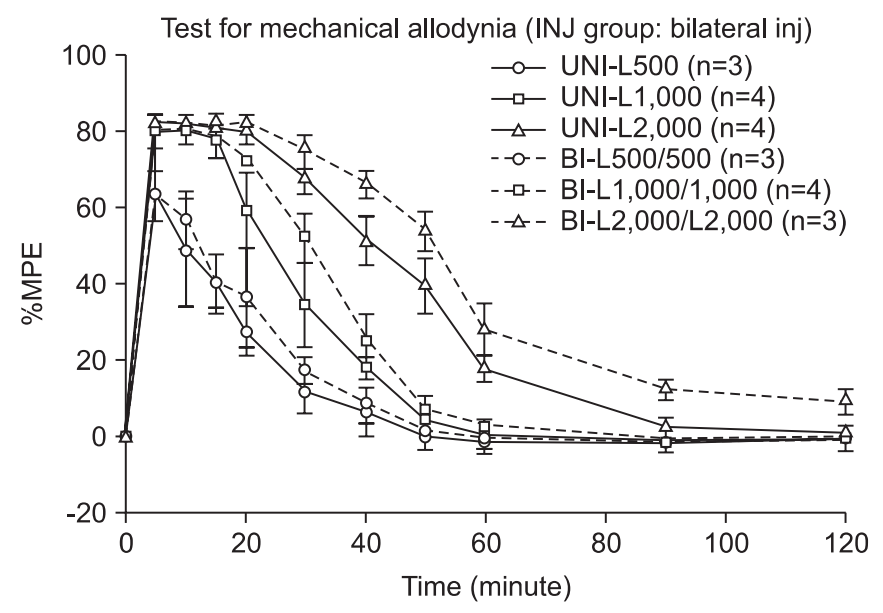

Fig. 4. (A) Comparison between ipsilateral and contralateral mechanical allodynia test of $0.5 \%, 1.0 \%$, and $2.0 \%$ lidocaine $100 \mu \mathrm{l}$ unilateral injection in the INJ group. IL: Ipsilateral, CL: Contralateral. (B) Comparison the results of ipsilateral mechanical allodynia test between unilateral and bilateral $0.5 \%, 1.0 \%$, and $2.0 \%$ lidocaine $100 \mu \mathrm{l}$ injection in the INJ group. UNI: Unilateral, BIL: Bilateral. (C) Comparison between the results of $0.5 \%, 1.0 \%$, and $2.0 \%$ lidocaine injection in the INJ and CATH groups.

animals is a measurement of altered pain threshold, induced by a controlled experimental setting. Pain threshold is affected by environmental stimuli and individual characteristics. Therefore, strict control of influencing factors can result in increased reliability of results. Surgery may affect the pain threshold of experimental animals. ${ }^{12}$ In this study, the body weight of rats showed a significant decrease at 24 hours after epidural catheterization; however, no decrease was observed in those without surgery. Fibrosis is also one of the influencing factors. Of particular importance, tube obstruction due to fibrosis precludes the experiment proceeding for a long period of time. Thus, catheterization is inappropriate for use in a 'chronic pain' animal study. Fibrosis develops from 2 days, and tube obstruction occurs frequently at 4 days after catheter implantation. ${ }^{13}$

Drug potential efficacy of $1.0 \%$ lidocaine $50 \mu$ injected 

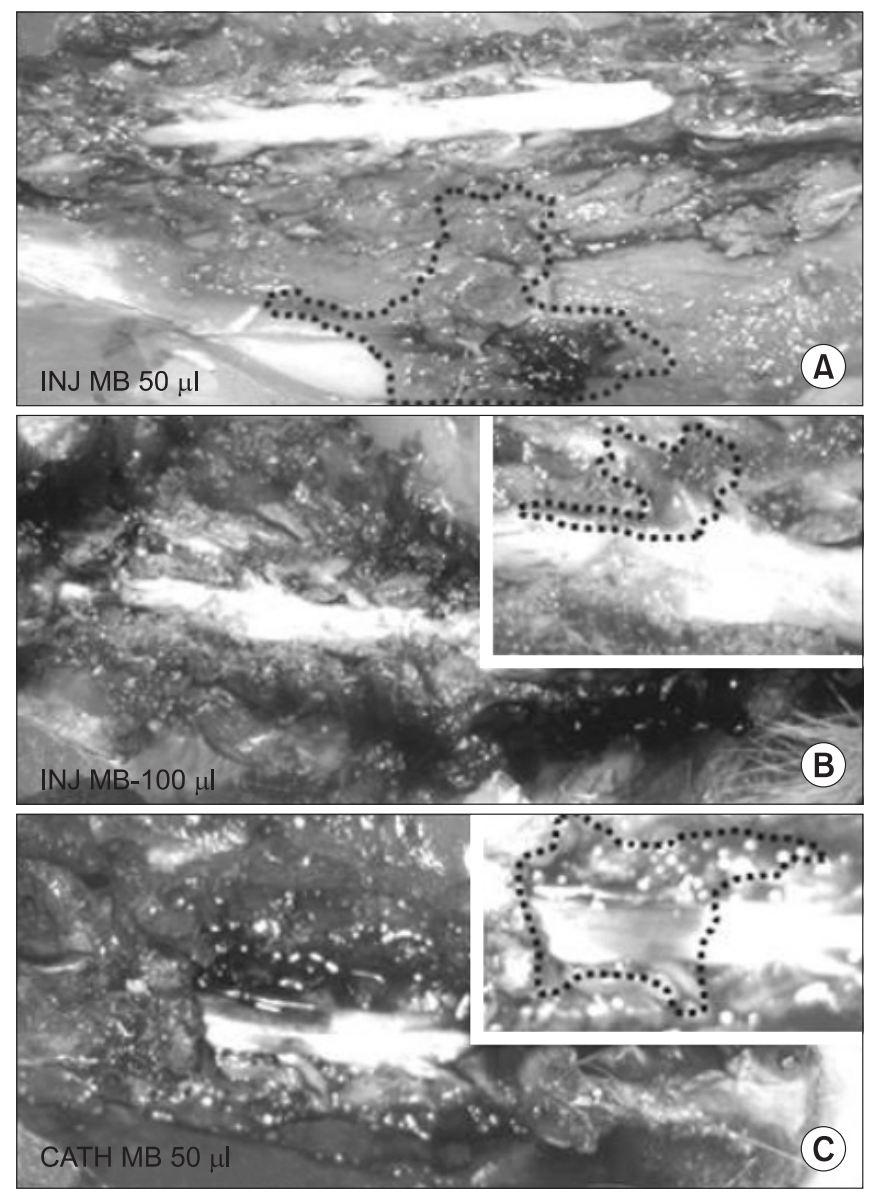

Fig. 5. Distribution of methylene blue (MB) after epidural injection of $50 \mu \mathrm{l}$ or $100 \mu \mathrm{l}$ injection: the distributed pattern is shown in dotted lines (A) $50 \mu \mathrm{l} \mathrm{MB}$ distribution in the INJ group. (B) $100 \mu \mathrm{l} \mathrm{MB}$ distribution in the INJ group. (C) $100 \mu \mathrm{l} \mathrm{MB}$ distribution in the CATH group.

through an epidural catheter does not statistically differ from that of $0.5 \%$ lidocaine $100 \mu \mathrm{l}$ injected through a unilateral or bilateral percutaneous transforaminal pathway. In addition, drug potential efficacy of $2.0 \%$ lidocaine $50 \mu \mathrm{l}$ injected through an epidural catheter also does not statistically differ from that of $1.0 \%$ lidocaine $100 \mu$ injected through a unilateral or bilateral percutaneous transforaminal pathway. Efficacy of a volume of $50 \mu$ injected through a percutaneous transforaminal pathway is indifferent to several lidocaine concentrations. The epidural distribution of methylene blue showed that lidocaine injected at a volume of $50 \mu \mathrm{l}$ did not sufficiently approach the epidural space in the vertebral foramen. These results suggest that a $50 \mu \mathrm{l}$ volume of liquid material is not able to pass through the narrow intervertebral foramen. ${ }^{14}$ However, this volume is thought to affect the distal spinal nerve of one or two spinal segments near the foramen. Despite that, the drug efficacies of three different concentrations did not differ statistically. The reason is that mechanical allodynia tests have insufficient sensitivity; it cannot detect each segmental sensory dysfunction. Lidocaine passed through the intervertebral foramen had different efficacies depending on concentration. However, the \%MPE during initial 20 minutes showed similar values. This may be a sealing effect, because the measuring range of von Frey filaments is too narrow for assessment of the changed thresholds.

Efficacies of $0.5 \%$ and $1.0 \%$ lidocaine $100 \mu \mathrm{l}$ did not differ significantly between unilateral and bilateral injection. Unilateral injection did not significantly affect the contralateral side. Grossly, the contralateral lower limb of rats injected unilaterally was observed to function normally. Results demonstrated the merits of this method of drug delivery method for anesthetization of only unilateral limbs.

This newly developed method for percutaneous transforaminal epidural drug injection has the following advantages. First, delivery failure and complications are rare. Therefore, assessment of the potential efficacy of the drug in animal studies of acute or chronic pain is more efficient than that by epidural catheterization. Second, the procedure is minimally invasive to the experimental animal. There is no insertion of foreign materials, no massive tissue incision, and no significant structural alteration, which may affect the pain threshold. Third, this method is easy to use by any experimenter. Decreased burden regarding time, labor, and cost is helpful for the purpose of focusing attention on the study as well as the aspects of the economical and serial plan.

However, this method also has the following disadvantages. First, using epidural catheterization, it is necessary to inject the same amount of drug bilaterally in order to show similar effectiveness. However, bilateral injection is easier and more time-effective than injection through an epidural catheter. Second, animals and experimenters are exposed to radiation. Quantitative measurement of exposure to radiation was not performed during this study. Five X-rays on average were projected per percutaneous injection, which were harmless to the rats. Third, insertion of the needle can cause mechanical injury to spinal nerves. The spinal nerve was placed on a caudal quarter of the intervertebral foramen on an X-ray lateral view. Because the diameter of the inserted needle is ap- 
proximately a quarter of the foramen, fine manipulation can protect against injury to spinal nerves.

The most important potential limitation of this study was that pathologic study for spinal nerve damage was not confirmed. We observed the animal's behavioral change only during recovery after injections. Using only the mechanical allodynia test for assessment of pain thresholds was also a considerable limitation. Other limitations included small experimental groups and no test for each spinal segment.

\section{CONCLUSION}

This newly proposed method is co-used with epidural catheterization, and is more simple and efficient than epidural catheterization in some animal studies for pain. It does not involve development of significant pain threshold alteration, and is able to anesthetize a unilateral limb through unilateral injection. A thoroughly planned additional study that makes up for some limitations is needed, and more completed method will be helpful for experimenters.

\section{REFERENCES}

1. van den Hoogen RH, Colpaert FC. Long term catheterization of the lumbar epidural space in rats. Pharmacol Biochem Behav 1981; 15: 515-516

2. Chapman CR, Casey KL, Dubner R, Foley KM, Gracely RH, Reading AE. Pain measurement: an overview. Pain 1985; 22: 1-31

3. Durant PA, Yaksh TL. Epidural injections of bupivacaine, morphine, fentanyl, lofentanil, and DADL in chronically implanted rats: a pharmacologic and pathologic study. Anesthesiology 1986; 64: 43-53

4. Casey WF, Burnell JC. Nerve root irritation from an epidural catheter. Anaesthesia 1982; 37: 697-698

5. Chaplan SR, Bach FW, Pogrel JW, Chung JM, Yaksh TL. Quantitative assessment of tactile allodynia in the rat paw. J Neurosci Methods 1994; 53: 55-63

6. Xu JJ, Walla BC, Diaz MF, Fuller GN, Gutstein HB. Intermittent lumbar puncture in rats: a novel method for the experimental study of opioid tolerance. Anesth Analg 2006; 103: 714-720

7. Eckel TS, Bartynski WS. Epidural steroid injections and selective nerve root blocks. Tech Vasc Interv Radiol 2009; 12: 11-21

8. Du Pen SL, Peterson DG, Williams A, Bogosian AJ. Infection during chronic epidural catheterization: diagnosis and treatment. Anesthesiology 1990; 73: 905-909

9. Kristensen JD, Post C, Gordh T Jr, Svensson BA. Spinal cord morphology and antinociception after chronic intrathecal administration of excitatory amino acid antagonists in the rat. Pain 1993; 54: 309-316

10. Tsang BK, He Z, Ma T, Ho IK, Eichhorn JH. Decreased paralysis and better motor coordination with microspinal versus PE10 intrathecal catheters in pain study rats. Anesth Analg 1997; 84: 591-594

11. Nagaro T. The histo-pathological changes in the epidural space and changes in the effect of anesthesia after long-term continuous epidural block. Masui 1986; 35: 227-235

12. Giebler RM, Scherer RU, Peters J. Incidence of neurologic complications related to thoracic epidural catheterization. Anesthesiology 1997; 86: 55-63

13. Nishiyama T. A rat model of chronic lumbar epidural catheterization. Can J Anaesth 1998; 45: 907-912

14. Furman MB, Mehta AR, Kim RE, Simin JI, Patel R, Lee TS, Reeves RS. Injectate volumes needed to reach specific landmarks in lumbar transforaminal epidural injections. PM R 2010; 2: 625-635 